\title{
A NEW CCD CAMERA AT THE MOLÉTAI OBSERVATORY
}

\author{
J. Zdanavičius and K. Zdanavičius \\ Institute of Theoretical Physics and Astronomy, Vilnius University, \\ Goštauto 12, Vilnius, LT-01108, Lithuania
}

Received November 5, 2003

\begin{abstract}
The results of the first testing of a new CCD camera of the Moletai Observatory are given. The linearity and the flat field corrections of good accuracy are determined by using shifted star field exposures.
\end{abstract}

Key words: methods: observational - techniques: CCD photometry, Vilnius photometric system

\section{INTRODUCTION}

In 2002 a VersArray 1300B CCD camera made by Princeton Instruments was bought for the Moletai Observatory. Here are the main parameters of the camera taken from the data sheet of the producer. The imaging array of the CCD chip has $1340 \times 1300$ pixels of $20 \times 20 \mu \mathrm{m}$ size. The linear area of the chip is $26.8 \times 26.0 \mathrm{~mm}$. The detector is a scientific-grade back-illuminated CCD chip with Unichrom UV-enhancement coating and liquid nitrogen cooling. The full well (single pixel) capacity is $200000 \mathrm{e}^{-}$. The quantum efficiency curve of the chip according to producer's information is shown in Figure 1. The $\mathrm{QE}$ at $300 \mathrm{~nm}$ is $\sim 40 \%$ and at $500-650 \mathrm{~nm}$ it is more than $90 \%$. The dark current is $\leq 1 \mathrm{e}^{-} / \mathrm{p} / \mathrm{hr}$

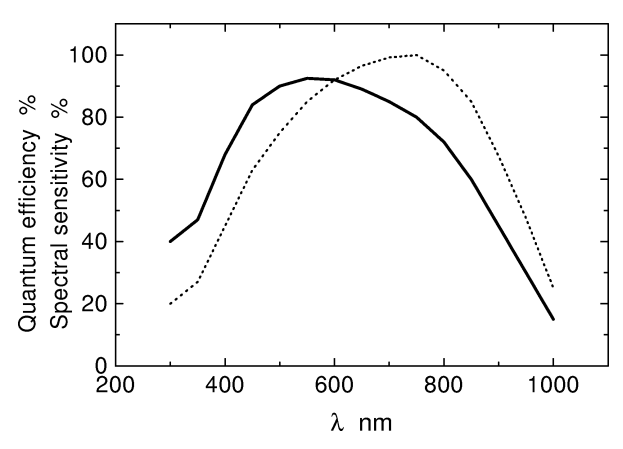

Fig. 1. Quantum efficiency (solid) and relative spectral sensitivity (dotted line) of the CCD. 
at $-120^{\circ} \mathrm{C}$. The system read noise

is $\leq 5 \mathrm{e}^{-}$rms and the full frame readout time is $18 \mathrm{~s}$ at a scan rate of $100 \mathrm{kHz}$. The dynamic range is 16 bits.

\section{LINEARITY TESTING}

The linearity of the CCD detector was tested in the laboratory. For this purpose we have illuminated the detector with a stable standard light source, placed in a special tube protecting the detector from outside light. Exposures of different length from a few seconds to 20 minutes to have different counts $N$ on CCD pixels have been taken. In this experiment we used only the central part of the detector to avoid the decrease of illumination intensity in the periphery. For each exposure time the numbers of counts per second $n$ (in ADU) were calculated. A gain of $2.3 \mathrm{e}^{-} /$cts was used. The numbers $n$, as a function of total number of detected counts $N$, are plotted in Figure 2 .

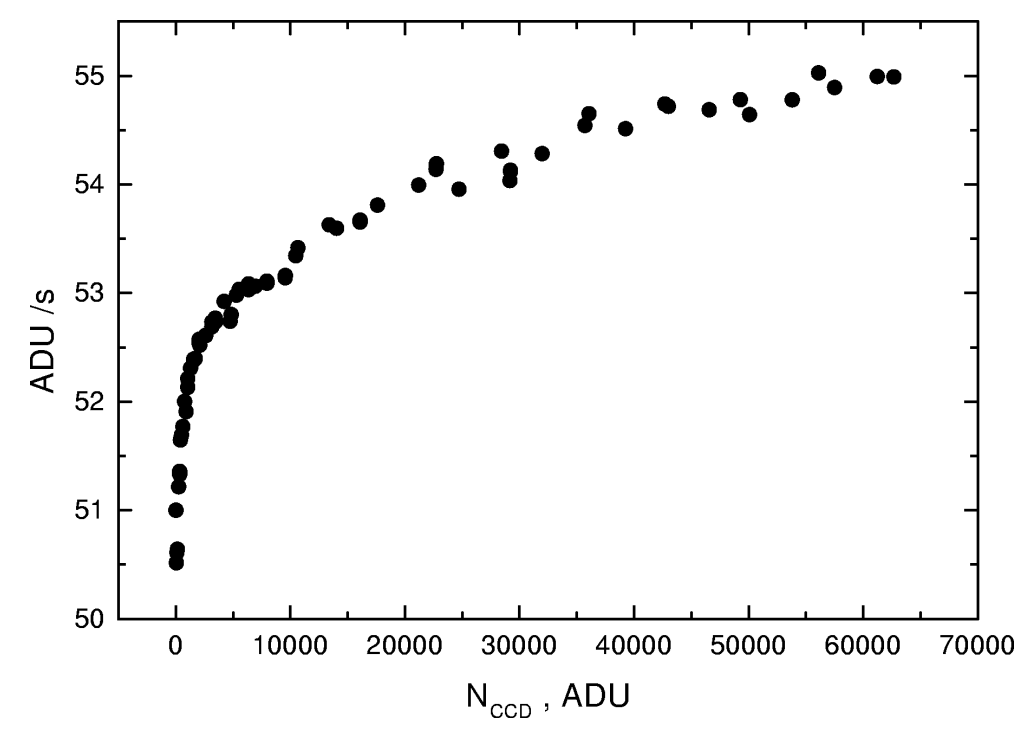

Fig. 2. Dependence of the number of counts (ADU) per second $n$ on the total number of the registered counts $N$.

We see that the mean number of counts per second $n$ grows with the increase of the total number of registered counts $N$. An especially large growth exists for small counts $N$, up to about 2000 counts. Deviations from the mean value $n=53 \mathrm{cts} / \mathrm{s}$ reach $\pm 4.5 \%$ at the extreme values of $N$. For the corrections for non-linearity two polynomials are used: one for the small values of counts $N$ up to 2610 and the second for the larger values. The residuals after the non-linearity corrections are 
much less than $1 \%$.

\section{OBSERVATIONS}

For observational testing the camera was installed in the Newtonian focus of the $35 / 51 \mathrm{~cm}$ Maksutov telescope of the Moletai Observatory.. The telescope has $51 \mathrm{~cm}$ diameter of the main mirror, $35 \mathrm{~cm}$ diameter of the meniscus lens, and the focal length ratio 3.5 (in the Newtonian focus). When used with the Maksutov telescope, each pixel of the CCD camera corresponds to 3.38 arcseconds. The field of view is $1.13 \times 1.22$ sq. degrees.

Observations in the Vilnius system have been done using the $U$ and $P$ glass filters, and $X, Y, Z, V$ and $S$ interference filters (60 $\mathrm{mm}$ diameter). The response functions of the camera are shown in Figure 3. The twilight flats were obtained in each filter.

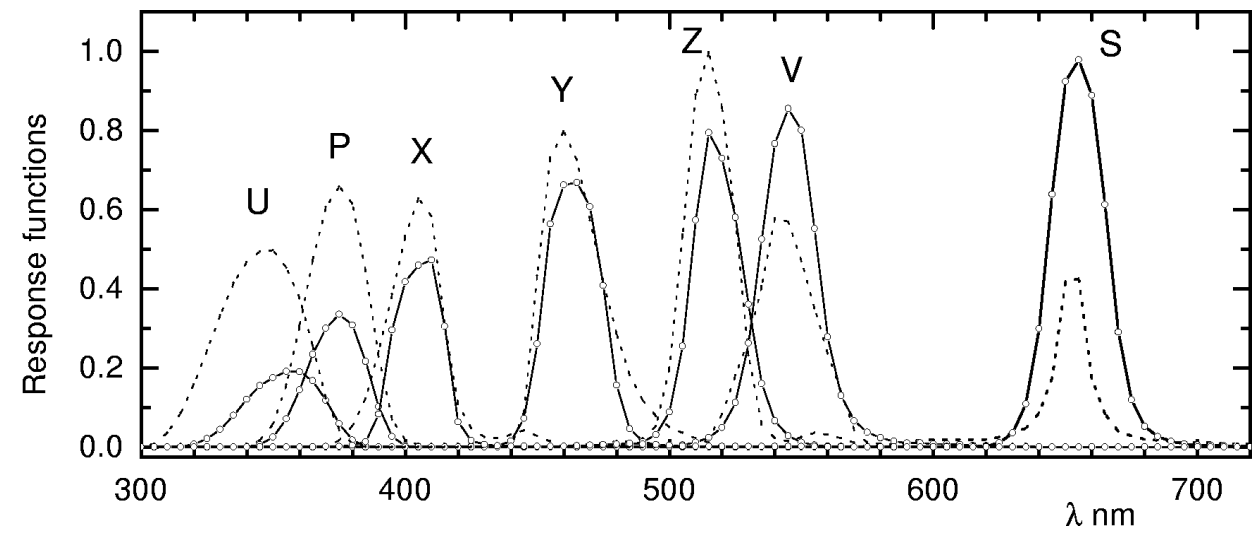

Fig. 3. Response functions of the instrumental CCD system (solid line) and the standard Vilnius system (dotted line). The standard functions are from Straižys (1992).

For determining the large-scale field correction we have obtained the exposures of star field with three different shifts. To correct for the linear tilt, the exposures using $V$ and $U$ filters were obtained by rotating the telescope by $180^{\circ}$. Magnitudes were obtained by aperture photometry using the standard IRAF program package. The linear equations for transformation of instrumental magnitudes and color indices to the standard Vilnius system were determined using the open cluster M67 stars (Laugalys et al. 2003) observed in the Vilnius system: 


$$
\begin{array}{lll}
U-V=-0.304+1.050(U-V)_{\mathrm{CCD}} & \pm 0.029 \\
P-V=-0.239+0.983(P-V)_{\mathrm{CCD}} & \pm 0.022 \\
X-V=0.830+1.054(X-V)_{\mathrm{CCD}} & \pm 0.017 \\
Y-V=0.396+0.934(Y-V)_{\mathrm{CCD}} & \pm 0.009 \\
Z-V=0.856+0.998(Z-V)_{\mathrm{CCD}} & \pm 0.008 \\
V-S=0.674+0.980(V-S)_{\mathrm{CCD}} & \pm 0.011
\end{array}
$$
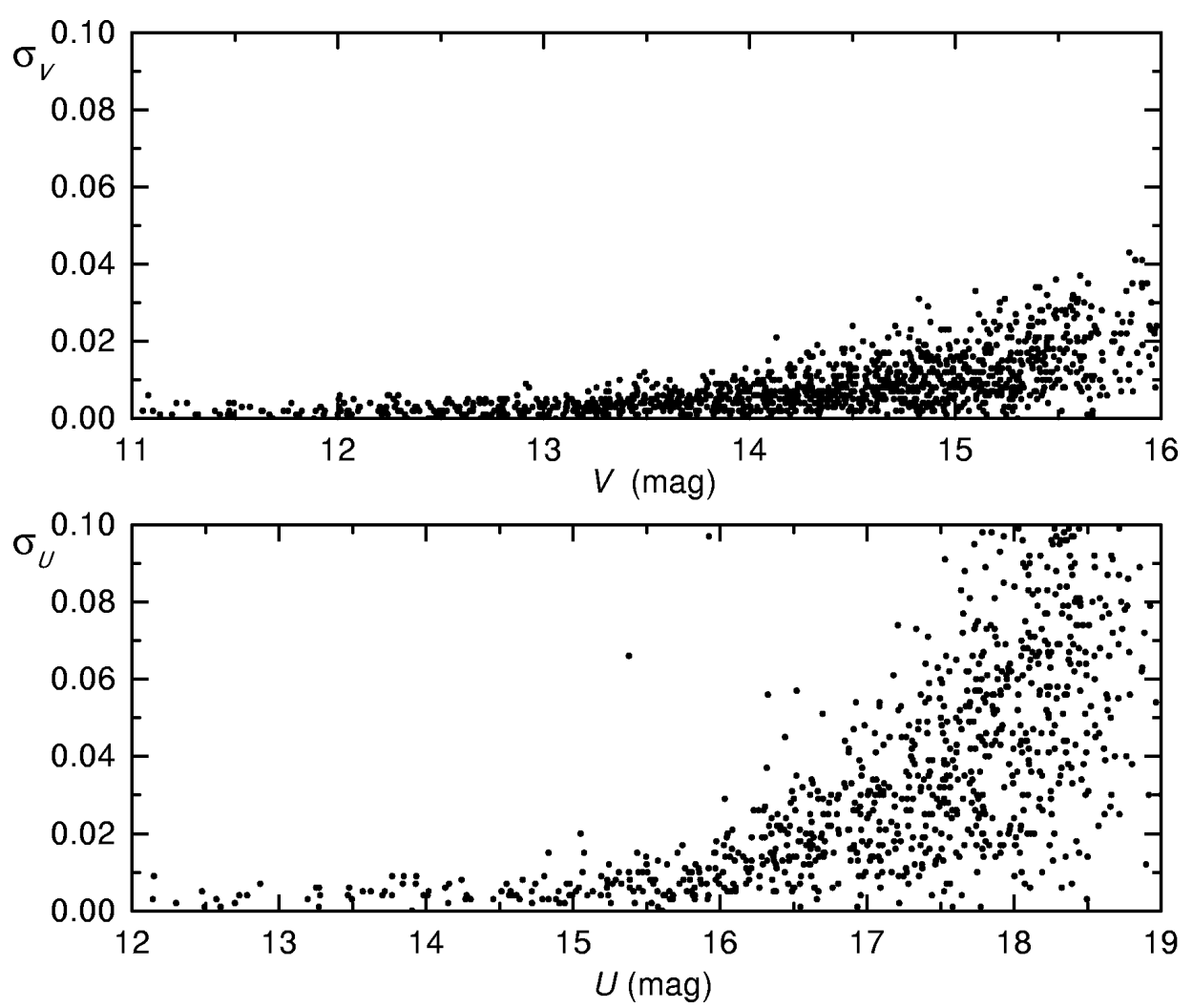

Fig. 4. The rms errors of the $U$ and $V$ magnitudes as a function of the star magnitude.

Exposures of several open clusters and dark cloud areas were also obtained. To evaluate the photometric accuracy, more than a thousand stars were measured in the three $U$ and three $V$ filter exposures of 
the area in the Camelopardalis dark cloud area. The three exposures in each filter were obtained with a small (about 50 pixels) shift. The exposure times in the $V$ filter were $4 \mathrm{~min}$ and in the $U$ filter $-30 \mathrm{~min}$. The deviations from the mean give the rms errors shown on Figure 4. For achieving the same accuracy in both filters we need exposure times in $U$ about ten times longer than in $V$.

\section{CONCLUSIONS}

(1) Due to the large field and good quality of stellar images the Maksutov telescope of the Moletai Observatory is a promising instrument for CCD photometry.

(2) For flat-fielding both the sky flats and the shifted exposures of a star field are necessary.

(3) For precise photometry the nonlinearity of the CCD response must be taken into account.

ACKNOWLEDGMENTS. We are grateful to V. Straižys for helpful suggestions. We also acknowledge the financial support of the Lithuanian Government for purchase of a CCD camera.

\section{REFERENCES}

Laugalys V., Boyle R. P., Kazlauskas A., Vrba F. J., Philip A. G. D., Straižys V. 2004, Baltic Astronomy, 13, in press

Straižys V. 1992, Multicolor Stellar Photometry, Pachart Publishing House, Tucson, Arizona 